\title{
ISLA NIMMT AN ERSTER FEIER FÜR NOBELPREISTRÄGERIN TU YOUYOU IN PEKING TEIL
}

\author{
Liebe Kolleginnen und Kollegen,
}
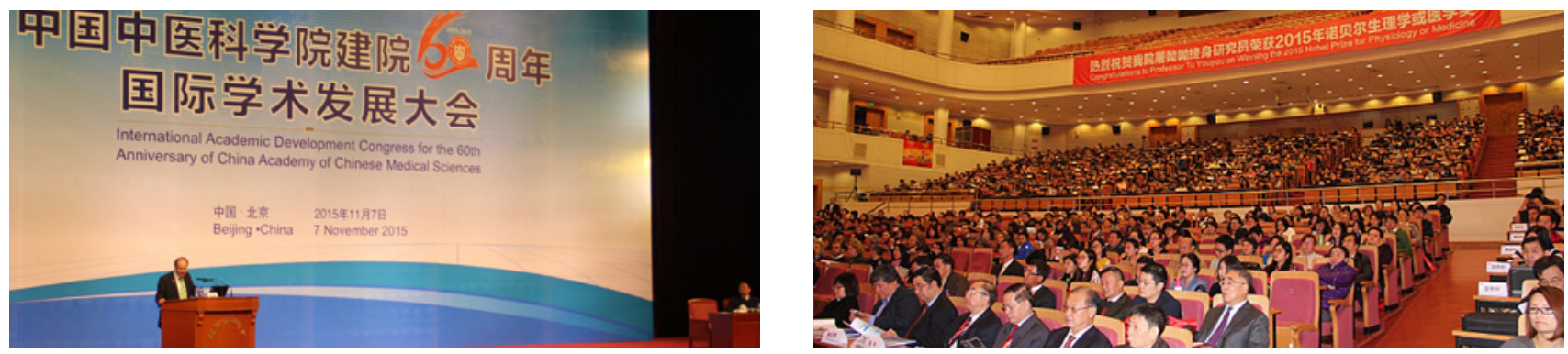

60-Jahr-Jubiläumsfeier der China Academy of Chinese Medical Sciences (7.11.2015) in Peking (900 TeilnehmerInnen).

am 7. November 2015 fand im größten Kongresszentrum in Peking die erste Feier für die Nobelpreisträgerin, Prof. Tu Youyou, im Rahmen des International Academic Development Congress for the $60^{\text {th }}$ anniversary of China Academy of Chinese Medical Sciences statt. Die ISLA war dabei vertreten durch Gerhard Litscher, den President for Science and Research, der gemeinsam mit derVizerektorin für Forschung und Internationales der Medizinischen Universität Graz, Frau Univ.-Prof. Dr. Dr.h.c. Irmgard Lippe, alsVertreter Europas sowohl Glückwünsche als auch einen Vortrag zur High-Tech Akupunktur übermitteln durfte.Weitere Informationen zur Nobelpreisträgerin finden Sie in einer eigenen Arbeit in diesem Heft.

Weitere wichtige ISLA-Veranstaltungen haben in Frankfurt, Laßnitzhöhe bei Graz und Bangkok (Thailand) im November und Dezember d.J. stattgefunden. Ein Highlight in diesem Zusammenhang war die Präsentation der Kombination von PRP (Platelet-Rich Plasma oder thrombo- zytenreiches Plasma) und Lasertherapie von weber medical, welche insbesondere in den USA bereits breitflächig bei orthopädischen Indikationen und im Bereich der regenerativen Medizin eingesetzt wird. Ein weiterer Teil der Veranstaltungen widmete sich der klinischen Anwendung der intravenösen Lasertherapie (aus der Perspektive der Mitochondrienmedizin), wobei neben den Grundlagen der Therapie auch aktuelle wissenschaftliche Daten präsentiert wurden. Die photodynamische Therapie wurde als vielversprechender neuer Ansatz in der Tumortherapie vorgestellt. In diesem Zusammenhang sind insbesondere die neuen Gelb- und Blaulaser entscheidende Weiterentwicklungen, um die Photosensitizer effektiv im Gewebe $\mathrm{zu}$ aktivieren.

Allen ISLA-Mitgliedern sowie allen Leserinnen und Lesern frohe Weihnachten sowie ein gesundes und erfolgreiches Jahr 2016!

Freundliche Grüße

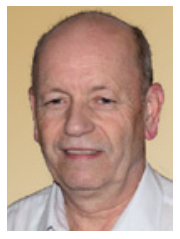

Dr. med. Dipl. Chem. Michael Weber

President for Medical and Clinical Applications der International Society for Medical Laser Applications (ISLAtranscontinental)

Klinik Neu-Mariahilf, Humboldtallee 10-12, D-37073 Göttingen, Tel. +49 5273/367 780

E-Mail: info@isla-laser.org, Internet:www.isla-laser.org

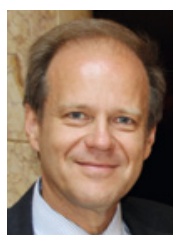

Univ.-Prof. Prof. h.c. DI Dr. techn.

Dr. scient. med.Gerhard Litscher

President for Science and Research der International Society

for Medical Laser Applications (ISLAtranscontinental)

Medizinische Universität Graz, Auenbruggerplatz 29,

A-8036 Graz, Tel: +43 316/385-13907, -83907

E-Mail:gerhard.litscher@medunigraz.at, Internet:http://litscher.info 